\title{
A NOTE ON THE FIXATION OF SPECIMENS FOR CELLOIDIN SECTIONING
}

\author{
BY
}

\author{
W. A. GRAY
}

LONDON

Fixation of the eye, without deformation of its component parts, is not easily obtained. This is due to the denseness of the outer coats and the loose attachment of the retina to the choroid. In addition, the contents of the eyeball are chiefly fluid.

There are two points of importance, first the fixative used and secondly the time of opening the eye. The usual procedure is to fix the eye for 24 hours in Zenker's solution or in saline containing five per cent. of formalin. To facilitate absorption of the fixing fluid the specimen is punctured with a needle. The eye is then frozen and opened with a median sagittal section. When the above method was employed detachment of the retina frequently resulted. Prolonged immersion (six weeks) in Müller's fluid gave no better results.

Despite the unfavourable opinion expressed by Heesch and others of alcohol as a fixative, this method gave the best sections. The vitreous was obtained almost complete, except for some shrinkage, between the ciliary body and the optic disc, away from the retina. Detachment of the retina was uncommon. It is essential that not more than 30 per cent. alcohol in distilled water should be used at first, and the strength of the alcohol is gradually increased by two per cent. up to absolute alcohol. The specimen is opened after the first 24 hours in absolute alcohol, when a small portion at the corneo-sclerotic junction is sliced off.

The complete method consists of the following:-

Stage 1.-Fixation for 24 hours in 30 per cent. alcohol in distilled water.

Stage 2.-Fixation in an alcohol series in which the percentage of alcohol is increased daily by 2 per cent. up to absolute.

Stage 3.-Opening of eyeball at corneo-sclerotic junction and further treatment in absolute alcohol for 24 hours.

Stage 4.-Embedding in celloidin in the usual fashion.

Three years' experience of this method has given me consistently good results. 